Cite this: Chem. Commun., 2014 50,8896

Received 6th May 2014

Accepted 14th June 2014

DOI: $10.1039 / \mathrm{c} 4 \mathrm{cc} 03387 \mathrm{f}$

www.rsc.org/chemcomm

\section{Near IR excitation of heavy atom free Bodipy photosensitizers through the intermediacy of upconverting nanoparticles $\dagger$}

\author{
Seda Demirel Topel, ${ }^{a}$ Gunseli Turgut $\mathrm{Cin}^{\mathrm{a}}$ and Engin U. Akkaya*bc
}

\begin{abstract}
Orthogonal dimers of Bodipy were recently shown to be efficient generators of singlet oxygen. However, these dyes require green light for excitation, which would be very quickly attenuated inside the mammalian tissues. We now demonstrate that when these dyes are covalently attached to UCNPs, near IR irradiation results in very efficient generation of singlet oxygen.
\end{abstract}

Recent years witnessed exponentially growing interest in a class of compounds known as Bodipy dyes (a.k.a., boradiazaindacenes or borondipyrrin dyes). ${ }^{1}$ It is remarkable that every single member of the tricyclic boradiazaindacene ring system, including the components of the difluoroboron bridge, can be derivatized, substituted or otherwise functionalized in a straightforward manner, yielding new spectral features, including red shifted absorption and emission, ${ }^{2}$ or large intersystem crossing rates. ${ }^{3}$ The latter is of particular importance if these dyes are to be proposed as potential singlet oxygen generators in a photodynamic therapeutic regime. Photodynamic therapy (PDT) is a non-invasive treatment modality for certain cancers and non-cancerous disease states. ${ }^{4}$ The treatment is based on the cytotoxicity of singlet oxygen at the region where it is generated, following sensitization of the dissolved oxygen by a dye with high intersystem crossing efficiency. There is an important caveat however; the dye has to have a strong absorption in the red or the near IR region of the spectrum, otherwise the excitation of the dye will be highly ineffective beyond the first few millimeters of the tissues. ${ }^{5}$ This is due to the fact that mammalian tissues are highly scattering and absorbing for light with wavelengths shorter than $630 \mathrm{~nm}^{6}$

Historically, porphyrins and derivatives were considered as the photosensitizers of choice for this purpose, but the extinction coefficients in the therapeutic window are not very high.

\footnotetext{
${ }^{a}$ Department of Chemistry, Faculty of Science, Akdeniz University, 07058, Antalya, Turkey

${ }^{b}$ UNAM-National Nanotechnology Research Center, Bilkent University, 06800,

Ankara, Turkey. E-mail: eua@fen.bilkent.edu.tr

${ }^{c}$ Department of Chemistry, Bilkent University, 06800, Ankara, Turkey

$\dagger$ Electronic supplementary information (ESI) available: Synthesis procedures and additional spectroscopic data. See DOI: 10.1039/c4cc03387f
}

Over the years, a number of classes of compounds were proposed as PDT photosensitizers, and within the last few years Bodipy dyes have been considered as viable alternatives to established examples. ${ }^{7}$ Although the parent dye absorbs in the middle of the visible region, it is possible to move the absorption band up to the near IR region with straightforward transformations. ${ }^{8}$

Parent (unmodified) Bodipy dyes typically are not effective photosensitizers. As a general rule, heavy atom substitution on the $\pi$-system results in enhanced intersystem crossing. ${ }^{9}$ However, the incorporation of heavy atoms for photosensitizers geared for photodynamic therapy would not be the optimal choice, because while the sensitization of molecular oxygen is very likely with this approach, increased dark toxicity is typically an undesired outcome. Dark toxicity limits the inherent selectivity of photodynamic therapy agents, which rests on the fact that the PDT agent is expected to be non-toxic unless/until it is at the right location for absorbing the light of excitation.

In our earlier work, ${ }^{10}$ followed by computational studies, ${ }^{11}$ and later corroborated by Zhang, ${ }^{12}$ it was demonstrated that orthogonal-Bodipy dyes, whether connected in a 8,2 - or a 8,8 -fashion, resulted in a very peculiar first excited state with tetraradicalic character and have very high propensity to undergo intersystem crossing. This finding was noteworthy, because in principle, the design is applicable to essentially all other chromophores, offering an alternative to heavy atom substitution as a safe bet for enhanced intersystem crossing (another approach for heavy-atom free photosensitization was developed by Zhao and co-workers ${ }^{13}$ ). While the detailed photophysical experimentation eliminating other possibilities, such as singlet fission, is awaited, one limitation expressed in the previous work ${ }^{10}$ was that the dimeric Bodipy dyes needed excitation at around $500 \mathrm{~nm}$, however for true photodynamic therapy potential, the excitation should be done in the therapeutic window, i.e., between 650 and $1000 \mathrm{~nm}$. With Bodipy dyes, conjugation extension by styryl modification has been shown to result in bathochromic shifts in the absorption band, but unfortunately, computational and experimental work showed that the excited state loses its tetraradicalic character in the longer wavelength absorbing derivatives 
of the dimeric chromophores, becoming much less efficient singlet oxygen generators.

In consideration of this, we investigated the possibility of using upconverting nanoparticles (UCNPs) as a solution to this problem. UCNPs can be excited with near IR irradiation and depending on the exact composition, emission at shorter wavelength regions of the spectrum is obtained. UCNPs have these remarkable properties due to the presence of trivalent lanthanide ions as dopants (as absorbers and emitters) in an inorganic matrix. ${ }^{14}$ This combination provides a multitude of metastable states with relatively long lifetimes. The main difference between two-photon absorbance (TPA) and upconversion is that, in upconversion, the virtual states of TPA are replaced by actual states, which are long-lived enough to be excited once more, under intense irradiation. The typical emitters are erbium, thulium and holmium ions due to the availability of equally spaced energy levels which would be optimal for absorption and energy transfer processes. ${ }^{15}$

The first goal of the present work was to synthesize appropriately derivatized orthogonal Bodipy dimers (Scheme 1). First of all, a positive control compound $\mathbf{1}$ was synthesized (ESI $\dagger$ ). This compound has two iodo substituents on the Bodipy core, ensuring good singlet oxygen generation upon excitation in the presence of dissolved molecular oxygen. Nagano ${ }^{16}$ and others ${ }^{17}$ demonstrated that such halogenated Bodipy compounds are indeed efficient photosensitizers.

As for the dimeric compounds, we have previously reported the synthesis of the dimeric parent compounds, ${ }^{10}$ but for covalent attachment, additional functionalization is needed. In the orthogonal dimer 2, the carboxylate anchor is placed on the meso-phenyl substituent. Formylation and the construction of the second Bodipy core in the usual manner yield the desired compound. The other dimer 3 carries the carboxylate in the 2-position of a Bodipy core, which is easily placed by a reaction with acrylic acid under palladium(II) catalysis (ESI $\dagger$ ). Absorption spectra of the Bodipy compounds show peaks in the

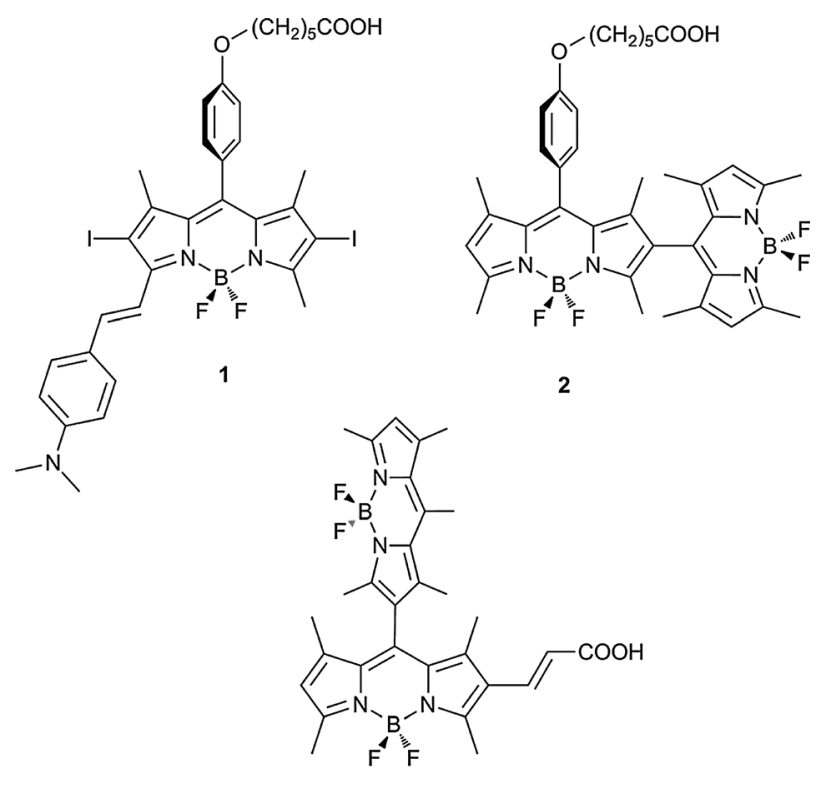

3

Scheme 1 Structures of the carboxy-functionalized sensitizers: styrylBodipy 1, orthogonal Bodipy dimers 2, and $\mathbf{3}$. visible region. Compound 1 shows a major peak at $638 \mathrm{~nm}$, red shifted due to dimethylaminostyryl conjugation. Dimers 2 and 3 show a major band peaking at 508 and $509 \mathrm{~nm}$, respectively, corresponding to $\mathrm{S}_{0}-\mathrm{S}_{1}$ transitions and the bands in the visible region are somewhat broadened. The peak shapes and positions are not affected by concentration changes, eliminating the possibility of any aggregation induced changes for spectral differences.

There are many successful techniques for the preparation of lanthanide-doped upconversion luminescence NPs, such as coprecipitation $^{18}$ and thermal decomposition. ${ }^{19}$ However, these methods involve organic solvent or high temperatures and usually require heat after treatment or produce toxic byproducts. ${ }^{20}$ There- $^{-}$ fore, in this work, we preferred to prepare upconverting nanoparticles by the solvothermal method ${ }^{21}$ which employs milder conditions and water based reaction medium leading to the formation of polyethyleneimine (PEI) covered nanoparticles for further functionalization with BODIPY derivatives. PEI is biocompatible $^{22}$ and PEI-nanoparticles are soluble in aqueous systems. It has been reported that free PEI can be toxic to cells, but when it is in particulate form, the hazardous effects are greatly decreased. ${ }^{23}$ When used for DNA delivery PEI was reportedly nontoxic at concentrations used in that study. ${ }^{24}$ In principle, the functional groups or water solubility conferring groups can be incorporated during synthesis or can be placed by post-synthetic modifications. We opted for in situ preparation which includes the addition of polyethyleneimine (PEI) groups during the nanoparticle synthesis. The synthesis procedure of the upconverting nanoparticles is presented in detail in ESI. $\dagger$ The size and morphology of the UCNPs were examined by TEM which shows an average size of $35 \mathrm{~nm}$ and this size distribution was also determined by DLS measurements. The TEM-EDAX spectrum shows that the rare earth cations were successfully doped into the UCNPs (Fig. 1). When excited using a near IR laser at $980 \mathrm{~nm}$

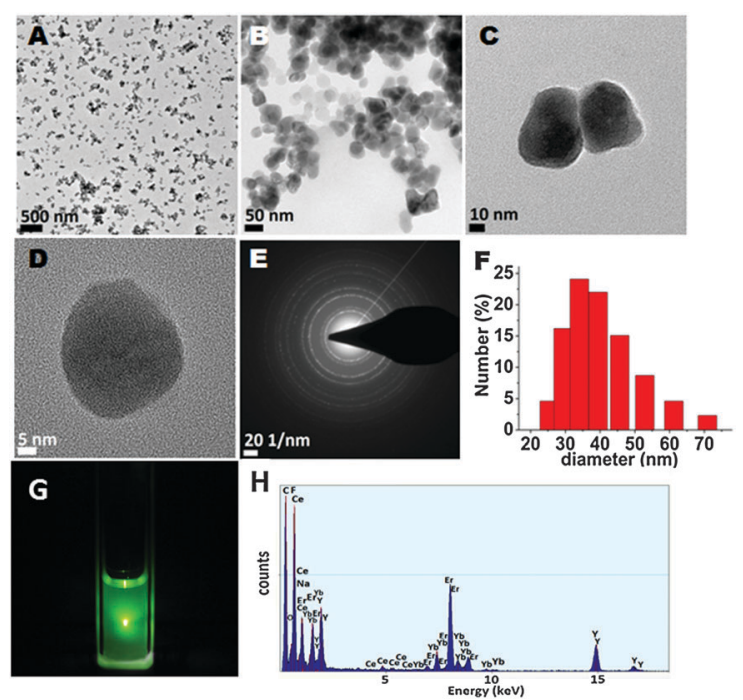

Fig. 1 TEM images of PEl-coated $\mathrm{NaYF}_{4}:\left(\mathrm{Yb}^{+3}, \mathrm{Er}^{+3}\right) / \mathrm{Ce}^{+3}$ nanoparticles (A-D), (E) SAED (selected area electron diffraction) pattern, (F) histogram of the particle size distribution from DLS measurements, (G) emission from UCNP solution in methanol $(1.0 \mathrm{mg} / 1.0 \mathrm{~mL})$ upon excitation with a $980 \mathrm{~nm}$ laser, $(H)$ TEM-EDAX spectrum of the PEI-coated UCNP. 
(fluence rate $50 \mathrm{~mW} \mathrm{~cm}^{-2}$ ), green emission from a solution containing these nanoparticles is clearly observed which is composed of two main peaks at 540 and $654 \mathrm{~nm}$ (Fig. S28, ESI, $\dagger$ and Fig. 1G). Further characterization at this stage was done by acquiring XPS and XRD data (Fig. S26 and S25, ESI, $\dagger$ respectively). XPS shows a particular peak at $402 \mathrm{eV}$, clearly belonging to $\mathrm{N}$ (1s) which corroborates that UCNP was covered by PEI in situ. The XRD spectrum also shows that UCNPs are in highly crystalline form including both hexagonal and cubic phases (Fig. S25, ESI $\dagger$ ).

For UCNP functionalization, the photosensitizers have to be prepared in an appropriately derivatized form. Orthogonal Bodipy dimer and styryl-Bodipy functionalization is shown in Scheme S1 $(\mathrm{ESI} \dagger)$. Detailed synthesis procedures are provided, here it will suffice to say that orthogonal Bodipy dimers with such remarkable excited state properties proved to be amenable to modifications leading to derivatives with conjugatable side groups. The next step was to conjugate these dyes onto the PEI coated UCNPs. This step proceeded smoothly, leading to sensitizer dye functionalized UCNPS (ESI, $\dagger$ Fig. S37) in the presence of DCC/DMAP coupling reagents.

BODIPY conjugated nanoparticles obtained in this way were first characterized physically. Following attachment of the BODIPY dyes to the UCNPs, no appreciable changes in the size were observed based on TEM analysis and also the TEM image (Fig. 2B) shows that there are fringes on the UCNPs, which prove that crystallinity is preserved, this also corroborated with the SAED image (Fig. 2C). The EF-TEM (Energy Filtered-TEM) map shows (Fig. 2, bottom three pictures) a halo of boron nuclei around the nanoparticles following the conjugation procedure.

On the other hand, the FTIR spectrum also supports covalent modification: there are new peaks at $1692-1686 \mathrm{~cm}^{-1}$ (1st amide bond, $\nu(\mathrm{C}=\mathrm{O})$ ), $1665-1656 \mathrm{~cm}^{-1}$ (2nd amide bond, $\nu(\mathrm{N}-\mathrm{H})$ ) and 1428-1422 $\mathrm{cm}^{-1}$ (3rd amide bond, $\nu(\mathrm{C}-\mathrm{N})$ ) attesting to the formation of the amide functionality between BODIPY dyes and UCNP. Before the conjugation reaction (i.e., PEI-covered UCNP) the nanoparticles show bands corresponding to the internal vibration of $\mathrm{NH}_{2}$ bands at 1630 and $1420 \mathrm{~cm}^{-1}$ and $\mathrm{CH}_{2}$ stretching at 2930 and $2850 \mathrm{~cm}^{-1}$ (Fig. S27, ESI $\dagger$ ).

The emission wavelength of UCNPs matched with the absorption wavelength of BODIPY dyes resulting in reasonable spectral overlap, and since the distance between the core and

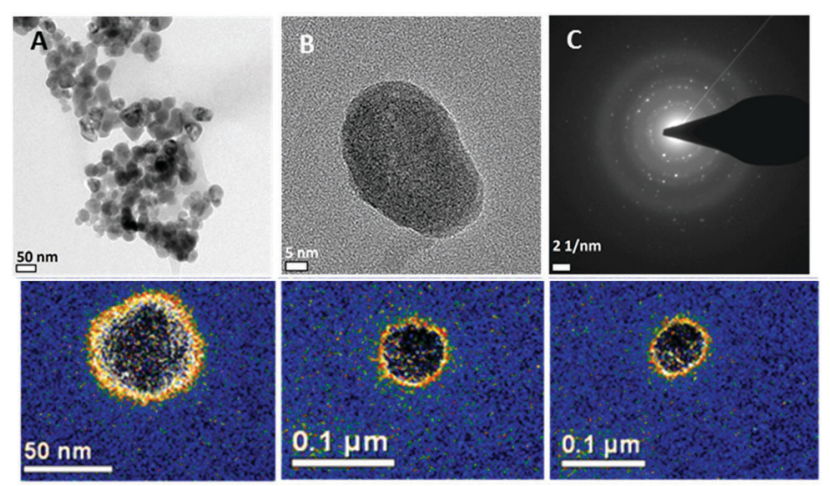

Fig. 2 TEM images (A, B), SAED (Selected Area Electron Diffraction) image (C) and EF-TEM boron mapping of BOD1-UCNP, BOD2-UCNP and BOD3-UCNP, left to right. Orange halo indicates boron nuclei (bottom three pictures). the dyes is short, efficient energy transfer is to be expected upon excitation of the UCNPs using a $980 \mathrm{~nm}$ laser (ESI, $\dagger$ Fig. S38).

Finally, we wanted to demonstrate the efficiency of singlet oxygen generation with specifically designed Bodipy dyes attached covalently to the UCNPs. The generation of singlet oxygen was monitored by using 1,3-diphenylisobenzofuran (DPBF) as a trap. ${ }^{25}$ When DPBF reacts with singlet oxygen, it is initially converted to its endoperoxide form, and then further transformed through ring opening into 1,2-dibenzoylbenzene, resulting in a decrease of the absorbance peak of DPBF. Therefore, the singlet oxygen production rate can be assessed following the change in the absorption of DPBF at the wavelength of absorption maximum ( $410 \mathrm{~nm}$ ) (Fig. S30-S32, ESI $\dagger$ ).

The procedure we followed for studying the relative rates of singlet oxygen generation with functionalized UCNPs (Fig. S39, ESI $\dagger$ ) is as follows: all BODIPY conjugated UCNPs were exposed to a NIR laser $\left(50 \mathrm{~mW} \mathrm{~cm}^{-2}\right)$ for $36 \mathrm{~min}$ (following 9 min dark incubation to eliminate any source for absorbance change due to a dark reaction). As expected, the adsorption of DPBF in the iodinated BOD1-UCNP $(\sim 26 \%)$ showed a decrease, but dimeric BOD2-UCNP showed a rapid decrease $(\sim 36 \%)$ during irradiation. The smallest change in the absorbance was observed in BOD3-UCNP $(\sim 23 \%)$. These results show that Bodipy dyes were effectively sensitized by the energy transfer from UCNPs. It is surprising that even a minimal overlap between the UCNP emission and the Bodipy emission in BOD2-UCNP is sufficient for effective sensitization. This is probably due to large extinction coefficients of the orthogonal Bodipy dyes and short distance between the dye and the particles.

In order to eliminate other possibilities for singlet oxygen generation, control experiments were carried out as follows: unmodified UCNPs and Bodipy dyes alone were subjected to near IR irradiation (980 nm) separately, no singlet oxygen generation (Fig. S33 and S34 ESI $\dagger$ ) was observed under either of these conditions.

In summary, we were able to show conclusively that UCNPconjugated orthogonal Bodipy dimers which require excitation at around $500 \mathrm{~nm}$ were successfully excited by a much more tissue penetrating $980 \mathrm{~nm}$ laser through the intermediacy of the upconversion process. Thus, Bodipy dimers with no heavy atoms can be considered as potential photosensitizers with practical utility when used in conjunction with UCNPs. Work towards making biocompatible nanoparticles with sensitizer functionality, aiming this particular goal, is in progress in our laboratory.

The authors gratefully acknowledge support from TUBITAK. The authors acknowledge the Akdeniz University Coordination Unit of Scientific Research Projects (project No. 2011.03.0121.017) for their financial support.

\section{Notes and references}

1 (a) A. Kamkaew, S. H. Lim, H. B. Lee, L. V. Kiew, L. Y. Chung and K. Burgess, Chem. Soc. Rev., 2013, 42, 77; (b) S. G. Awuah and Y. You, RSC Adv., 2012, 2, 11169; (c) G. Ulrich, R. Ziessel and A. Harriman, Angew. Chem., Int. Ed., 2008, 47, 1184.

2 (a) T. Mizuno, K. Umezawa, Y. Shondo, D. Citterio, K. Oka and K. Suzuki, J. Fluoresc., 2013, 23, 1007; (b) A. Poirel, A. De Nicola and R. Ziessel, J. Org. Chem., 2011, 76, 4489; (c) S. Kolemen, Y. Cakmak, S. Erten Ela, Y. Altay, J. Brendel, M. Thelakkat and E. U. Akkaya, 
Org. Lett., 2010, 12, 3812; (d) Z. Dost, S. Atilgan and E. U. Akkaya, Tetrahedron, 2006, 62, 8484.

3 M. J. Ortiz, I. Garcia Moreno, A. R. Agarrabeita, G. Duran Sampedro, A. Costela, R. Sastre, F. Lopez Arbeloa, J. Banuelos Prietoand and I. Lopez Arbeloa, Phys. Chem. Chem. Phys., 2010, 12, 7804.

4 (a) D. E. J. G. J. Dolmans, D. Fukumura and R. K. Jain, Nat. Rev. Cancer, 2003, 3, 380; (b) Z. Huang, Technol. Cancer Res. Treat., 2005, 4, 283.

5 K. Plaetzer, B. Krammer, J. Berlanda, F. Berr and T. Kiesslich, Lasers Med. Sci., 2009, 24, 259.

6 T. Vo-Dinh, Editor-in-Chief, Biomedical Photonics Handbook, CRC Press, Boca Raton, Florida, 2003.

7 (a) Y. Cakmak, T. Nalbantoglu, T. Durgut and E. U. Akkaya, Tetrahedron Lett., 2014, 5, 538; (b) S. Erbas Cakmak and E. U. Akkaya, Angew. Chem., Int. Ed., 2013, 52, 11364; (c) S. Erbas, M. Kocakusakogullari and E. U. Akkaya, Chem. Commun., 2009, 4956.

8 S. Ozlem and E. U. Akkaya, J. Am. Chem. Soc., 2009, 131, 48.

9 N. Adarsh, R. R. Avirah and D. Ramaiah, Org. Lett., 2010, 12, 5720 .

10 Y. Cakmak, S. Kolemen, S. Duman, Y. Dede, Y. Dolen, B. Kilic, Z. Kostereli, L. Tatar Yildirim, A. L. Dogan, D. Guc and E. U. Akkaya, Angew. Chem., Int. Ed., 2011, 50, 11937.

11 S. Duman, Y. Cakmak, S. Kolemen, E. U. Akkaya and Y. Dede, J. Org. Chem., 2012, 77, 4516.

12 X. F. Zhang and X. Yang, J. Phys. Chem. B, 2013, 117, 9050.

13 L. Huang, X. R. Yui, W. H. Wu and J. Z. Zhao, Org. Lett., 2012, 14, 2594.
14 (a) F. Wang, D. Banerjee, Y. Liu, X. Chen and X. Liu, Analyst, 2010, 135, 1839; (b) H. Schäfer and M. Haase, Angew. Chem., Int. Ed., 2011, 50, 5808.

15 H. X. Mai, Y. W. Zhang, R. Si, Z. G. Yan, L. D. Sun, L. P. You and C. H. Yan, J. Am. Chem. Soc., 2006, 128, 6426.

16 T. Yogo, Y. Urano, Y. Ishitsuka, F. Maniwa and T. Nagano, J. Am. Chem. Soc., 2005, 127, 12162.

17 (a) C. S. Zhang, J. Z. Zhao, S. Wu, Z. L. Wang, W. H. Wu, J. Ma, S. Guo and L. Huang, J. Am. Chem. Soc., 2013, 135, 10566-10578; (b) S. Atilgan, Z. Ekmekci, A. L. Dogan, D. Guc and E. U. Akkaya, Chem. Commun., 2006, 4398.

18 (a) S. Heer, K. Kömpe and H. U. Gudel, Adv. Mater., 2004, 16, 2102; (b) J. H. Zeng, J. Su, Z. H. Li, X. Ja and Y. D. Li, Adv. Mater., 2005, 2119.

19 (a) J. C. Boyer, F. Vetrone, L. A. Cuccia and J. A. Capobianeo, J. Am. Chem. Soc., 2006, 128, 7444; (b) H. X. Mail, Y. W. Zhang and R. Si, J. Am. Chem. Soc., 2006, 128, 6426.

20 F. Wang and X. Liu, Chem. Soc. Rev., 2009, 38, 976.

21 (a) X. F. Yu, Z. Sun, M. Li, Y. Xiang, Q. Q. Wang, F. Tang, Y. Wu, Z. Cao and W. Li, Biomaterials, 2010, 31, 8724; (b) X. Yu, M. Li, M. Xie, L. Chen and Q. Wang, Nano Res., 2010, 3, 51.

22 F. Wang, D. K. Chatterjee, Z. Li, Y. Zhang, X. Fan and M. Wang, Nanotechnology, 2006, 17, 5786.

23 W. T. Godbey, K. K. Wu and A. Mikos, J. Controlled Release, 1999, 60, 149.

24 R. C. Lambert, Y. Maulet, J. L. Dupont, S. Mykita, P. Craig, S. Volsen and A. Feltz, Mol. Cell. Neurosci., 1996, 7, 239.

25 S. Erbas Cakmak, O. A. Bozdemir, Y. Cakmak and E. U. Akkaya, Chem. Sci., 2013, 4, 858. 